\title{
Artículos
}

\section{La gestión de documentos electrónicos: requerimientos funcionales}

\author{
Por David Gómez Domínguez, Antonio Ángel Ruiz Rodríguez y Eduardo Peis Redondo
}

\begin{abstract}
David Gómez Domínguez, premio nacional fin de carrera, es licenciado en biblioteconomía y documentación por la Universidad de Granada. Actualmente es becario de investigación del Ministerio de Educación, Cultura y Deporte en la Facultad de Biblioteconomía y Documentación de la citada universidad, donde está realizando su tesis doctoral en sistemas de gestión de archivos electrónicos y trabaja para distintos proyectos de investigación e innovación docente relacionados con el documento electrónico.
\end{abstract}

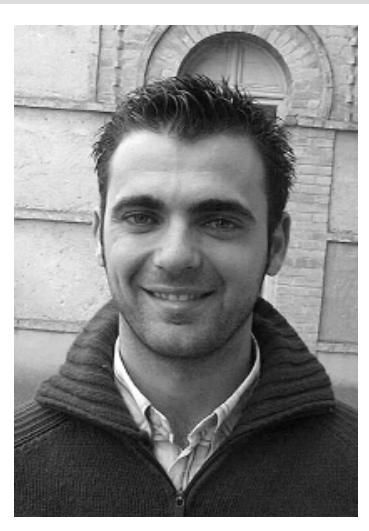

Resumen: Tras describir brevemente el cambio que ha ocasionado la aparición de los documentos electrónicos en el ámbito de la gestión, nos centramos en el diseño e implementación de sistemas de gestión de documentos electrónicos, y más concretamente en el establecimiento de requerimientos funcionales en esta materia. A continuación se analizan algunas de las listas de requerimientos establecidas haciendo especial hincapié en la especificación europea MoReq. Por último, concluimos ofreciendo un listado con las grandes áreas de establecimiento de este tipo de requerimientos y una tabla comparativa de cada una de estas listas con respecto a MoReq.

Palabras clave: Documentos electrónicos, Gestión de documentos, Sistemas de gestión de documentos electrónicos, Requerimientos funcionales, Listas de requerimientos.

\section{Title: Electronic records management: functional requirements}

Abstract: After briefly describing how the emergence of electronic records has brought changes to records management, we focus on the design and implementation of electronic records management systems, and specifically on the establishment of functional requirements. A number of functional requirements lists are then analysed, with particular attention given to the European MoReq specification. To conclude there is a listing of broad areas in which this type of requirement is being established, as well as a table comparing each one of these lists with MoReq.

Eduardo Peis Redondo, doctor en documentación, es profesor de la Universidad de Granada desde 1991. Especializado en la investigación sobre metalenguajes normalizados como sgml/xml y su aplicación a los servicios de in formación. La confluencia entre la docencia y dicha línea de investigación ha producido numerosos trabajos (publicados en las principales revistas internacionales del área), alguno de los cuales se centra en el papel de los modelos de metadatos en el diseño de los modernos sistemas de gestión de archivos electrónicos. La representación de la información en la web, con xml/xsl, y la descripción de recursos, con rdf, completan su actividad investigadora, ahora especialmente dirigida hacia la futura web semántica.

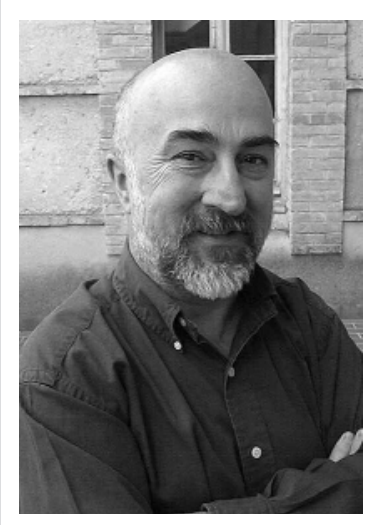

Antonio Ángel Ruiz Rodríguez es catedrático en la Universidad de Granada, ejerciendo la docencia desde 1978 siempre relacionada con los archivos, con especial atención a archivos personales y fotográficos. En los últimos años desarrolla una línea de investigación centrada en el documento electrónico y la recuperación de información, con aportaciones científicas y dirección de tesis doctorales que relacionan y aplican las normas archivísticas en la gestión de documentos e imágenes como es el caso de la descripción EAD (Encoded Archival Description).

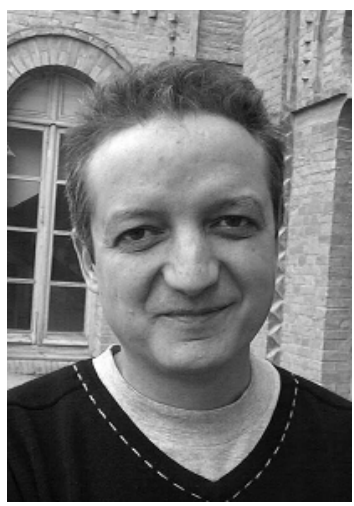

Keywords: Electronic records, Records management, Electronic records management systems, Functional requirements, Requirement lists.

Gómez Domínguez, David; Ruiz, Rodríguez, Antonio Ángel; Peis Redondo, Eduardo. "La gestión de documentos electrónicos: requerimientos funcionales". En: El profesionalde la información, 2003, marzoabril, v. 12, n. 2, pp. 88-98. 


\section{Introducción}

La revolución de la tecnología informática y de los sistemas de información ha cambiado drásticamente la manera en que los gobiernos, organizaciones e individuos comunican y realizan sus actividades diarias; es decir, la forma en que crean y mantienen sus documentos. Muchos de los documentos tradicionalmente creados y conservados en papel ahora lo son en forma electrónica. Éstos deben ser conservados permanentemente por motivos administrativos, legales o culturales ya que proporcionan evidencia de políticas, procedimientos, acciones o decisiones. Sin embargo, la obsolescencia tecnológica, la fragilidad de los soportes de almacenamiento y la manipulabilidad de los sistemas informáticos ponen en entredicho nuestra capacidad de garantizar tanto la conservación a largo plazo como la autenticidad de los mismos.

\section{«Los requerimientos funciona- les describen lo que debe ha- cer el sistema de gestión de documentos; qué debe hacer, pero no cómo Ilevarlo a cabo»}

Una de las principales líneas de investigación que se está llevando a cabo en nuestra comunidad científica, junto con otras tantas en este sentido, es el diseño de sistemas de gestión de documentos electrónicos (sgde). En este contexto, el término sistema se usa en su sentido más amplio para describir la misión organizativa, los procesos comerciales, las políticas, los procedimientos, las prácticas y los mecanismos humanos y automatizados para conseguir los fines deseados, que en este caso consisten en una gestión de registros fiable. Para conseguirlo en el entorno tecnológico se está proponiendo la identificación de un conjunto de requerimientos que han de cumplir los sgdes (1).

El proyecto de la Universidad de Pittsburgh, pionero en este sentido, estableció un conjunto de requerimientos funcionales para la gestión de documentos distribuidos en tres niveles:

-Organización.

—Sistema de gestión de documentos.

-Documento.

A su vez cada uno de ellos se subdividía en cinco categorías: organización consciente, sgde fiable, documentos capturados, documentos mantenidos y disponibles. Dentro de estas categorías se establecieron veinte requerimientos identificados por la ley, las normas y las mejores prácticas, como propiedades fundamentales de los documentos evidenciales (2).
A partir de ese momento han sido numerosas las listas de requerimientos para sgde que se han ido produciendo. Algunas de ellas son las definidas por:

-El Departamento de Defensa $(D o D)$ de EUA (3).

- Los archivos nacionales de Australia, Noruega y Canadá $(4,5,6)$.

- Los archivos estatales de Victoria (Australia), Nueva York, Delaware y Kansas (7, 8, 9, 10).

- Las universidades de Indiana y British Columbia $(11,12)$.

Actualmente ha sido desarrollado a nivel europeo y gestionado por el programa IDA (Interchange of $D a$ ta between Administrations) un modelo de requerimientos funcionales en materia de gestión de documentos electrónicos; nos estamos refiriendo a la especificación MoReq — Model Requirements for the Management of Electronic Records- (13).

Otra cuestión a analizar en relación con los sgde es dónde se va a integrar esta funcionalidad de gestión. Se plantean varias posibilidades:

-Integrarla dentro del sistema de gestión de información de la organización.

- Gestionar los documentos en un sistema completamente separado.

—Una combinación de ambos enfoques.

Hasta el momento no hay acuerdo en este sentido debido, en gran medida, a que no se han realizado los tests significativos de costes y rentabilidad de cada una de las alternativas planteadas. Ahora bien, la metodología para el diseño de sgdes queda recogida en normas globales que están desarrollando países como:

-Australia ( $A S 4390$ en un principio y actualmente $A S I S O$ 15489).

-EUA (36 CFR Part 1234: Electronic records management, elaborada por la Nara, National Archives and Records Administration).

-Reino Unido (programa Eros, Electronic Records from Office Systems. Management, appraisal and preservation of electronic records, llevado a cabo por el PRO, Public Record Office).

Para normalizar esta situación a nivel europeo, la $I S O$ ha propuesto un estándar de gestión de documentos de aplicación internacional. Se trata de la norma ISO 15489 sobre gestión de documentos lanzada en su primera edición en septiembre de 2001 por el Technical Committee ISO/TC 46, Information and Documentation, Subcommittee SC 11, Archives/Records Management. Se desarrolló en respuesta al acuerdo general 
entre los países miembros de la ISO, para normalizar a nivel internacional la gestión de registros tomando como base las normas australianas $A S 4390^{1}$. Fue diseñada para asegurar una protección apropiada a todos los documentos, y poder recuperar, de forma más eficaz, la evidencia e información que contienen éstos usando procedimientos normalizados (14).

Esta metodología, que actualmente queda recogida en la ISO 15489, incluye las siguientes fases:

-A: investigación preliminar.

-B: análisis de las actividades comerciales.

- C: identificación de las necesidades en materia de gestión de documentos.

—D: evaluación de los sistemas existentes.

-E: identificación de estrategias para satisfacer los requerimientos en materia de gestión de documentos.

-F: diseño de un sgde.

- G: implementación de un sgde.

-H: revisión posterior a la implementación.

En pocas palabras, y como síntesis, decir que un sgde establecido debe asegurar (15):

-Que los documentos electrónicos puedan mantenerse accesibles y legibles a lo largo del tiempo.

-Que mediante su contenido, contexto y estructura den evidencia fidedigna de las actividades de su creador, salvaguardándolos de las posibles manipulaciones y alteraciones.

-Que los documentos vitales sean identificados y conservados de la mejor forma.

Ahora bien ¿cómo identificamos y establecemos estos requerimientos funcionales?

Como hemos podido comprobar uno de los primeros pasos a la hora de diseñar e implementar un sgde lo constituye el establecimiento de las necesidades o requerimientos funcionales que debe cumplir el sistema en relación con el entorno comercial, legal, administrativo y social de la organización. Por tanto, se derivan de un análisis sistemático tanto de las necesidades comerciales internas de la organización como de las regulaciones externas impuestas por las leyes y normas nacionales e internacionales, las mejores prácticas de gestión de documentos y las expectativas de la sociedad o comunidad en general. En definitiva, los requerimientos funcionales describen lo que debe hacer el sgde; qué debe hacer, pero no cómo llevarlo a cabo.
Así, de forma general, podemos deducir que existen dos tipos de requisitos funcionales (15):

-Internos: implicados por el entorno comercial o sociopolítico en el cual opera la organización.

-Externos: definidos explícitamente en leyes, normativas y otros instrumentos de autoridad.

El objetivo que se persigue con el establecimiento de los requerimientos de una organización no es otro que garantizar y guardar la evidencia de sus actividades.

\section{«Los usuarios que tomen como base MoReq para diseñar su sgde podrán añadir requeri- mientos específicos adiciona- les o eliminar alguno de sus aspectos opcionales»}

A continuación recogemos los pasos a seguir para establecer el listado de requerimientos funcionales que debe cumplir el sgde de una organización:

- En primer lugar debemos llevar a cabo una investigación preliminar que nos proporcione un conocimiento general de la organización y de los contextos administrativo, legal, comercial y social en los que opera (figura 1).

- Con posterioridad realizaremos un análisis de las funciones, actividades y transacciones de la organización e identificaremos sus procesos comerciales, específicamente la relación entre los negocios de la organización y los documentos resultantes de los mismos, pues son los productos y evidencia de sus actividades (figura 2).

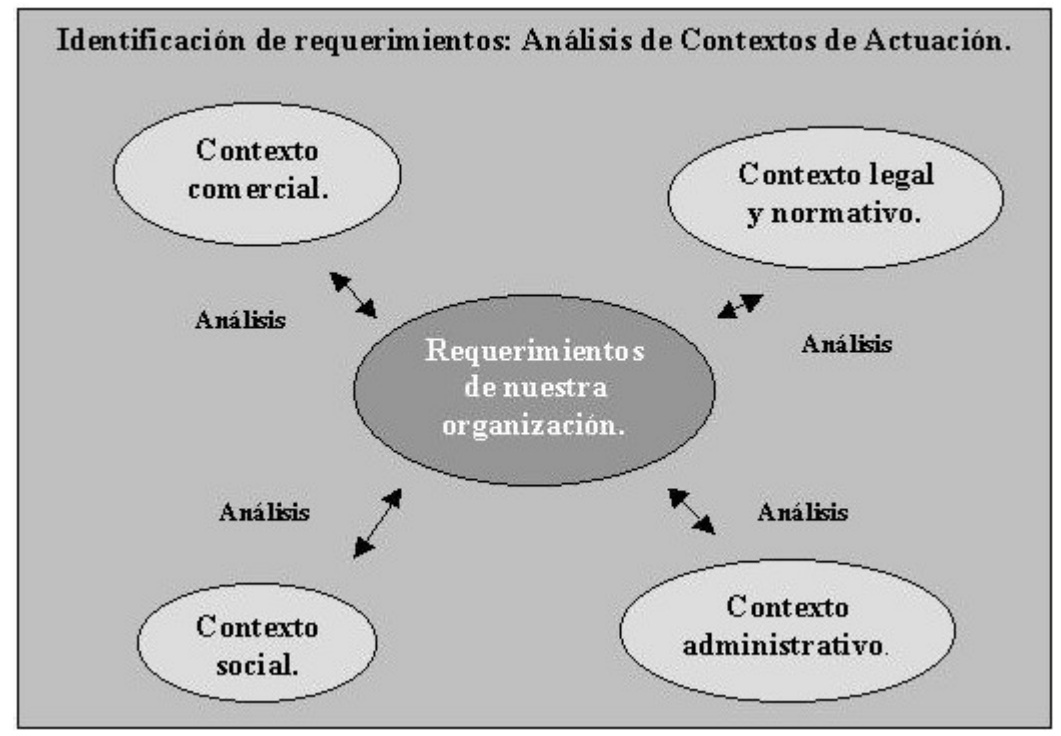

Figura 1 


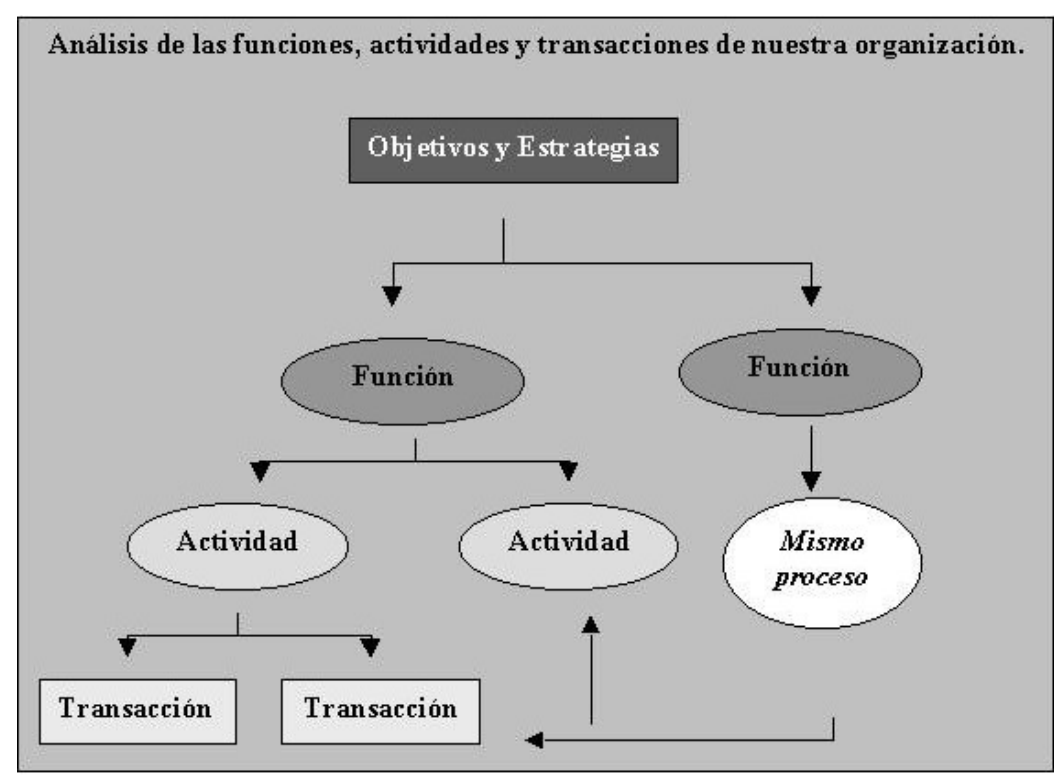

Figura 2

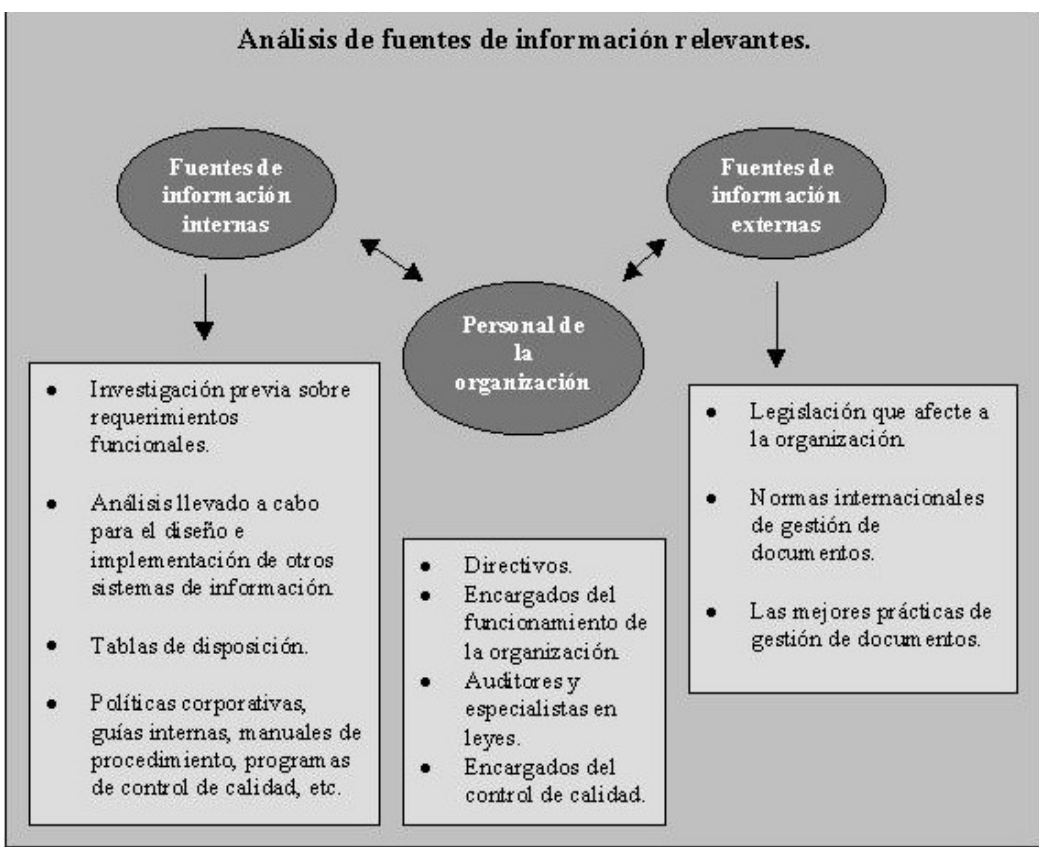

Figura 3

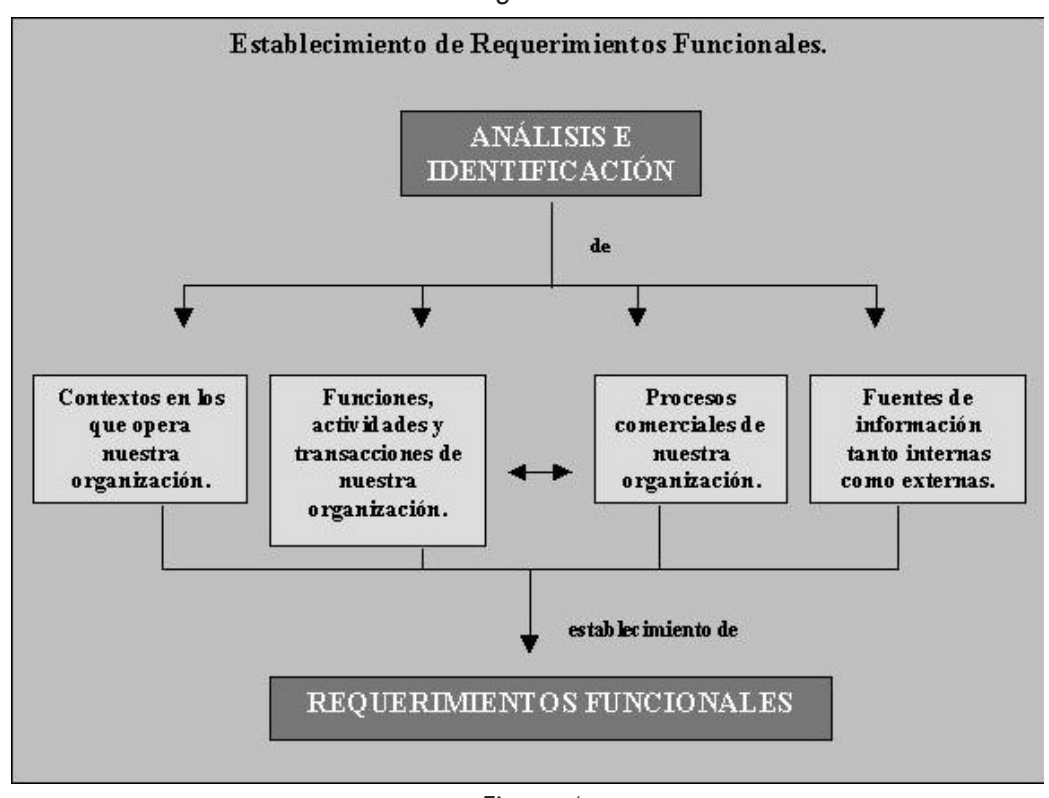

Figura 4
-Por último, llevaremos a cabo un análisis de fuentes de información relevantes (figura 3).

Por tanto, y resumiendo, el proceso completo para la identificación y establecimiento de requerimientos funcionales es el que se observa en la figura 4.

Una vez finalizadas estas fases, y sobre la base de los resultados obtenidos, estamos en condiciones ya de establecer el listado de requerimientos que debe cumplir el sgde de la organización.

\section{La especificación MoReq}

Como hemos comentado con anterioridad, ha sido creada a nivel europeo y gestionada por el programa IDA. La necesidad de desarrollarla fue articulada por el DLM-Forum en 1996 como uno de los 10 puntos de acción que surgieron del encuentro. No obstante, el proyecto fue lanzado tres años más tarde, en 1999, el trabajo comenzó en el año 2000 y concluyó a comienzos del 2001. Fue llevado a cabo por un pequeño equipo de asesores ${ }^{2}$ especialistas de Cornwell Affiliates plc. Consultants in Management and IT ${ }^{3}$, apoyados o respaldados siempre por las orientaciones de un grupo de expertos ${ }^{4}$ de varios países y unas organizaciones de validación tanto privadas como públicas (tabla 1).

Aunque es una especificación diseñada con la experiencia de varios países europeos, intenta ser compatible no sólo con los países de la Unión Europea, sino también con todos aquellos donde se empleen sistemas para gestionar la información electrónica. Está pensada para ser completamente genérica. Por esta razón, pretende abarcar un amplio rango de necesidades con el objetivo de servir como modelo. No todos los requerimientos pueden ser aplicados en algunos entornos ya que cada país tiene sus propias tradiciones, visiones y normativas sobre gestión de documentos. Dada esta característica, los usuarios que tomen como base MoReq para diseñar su sgde podrán añadir aspectos específicos adicionales o eliminar alguno de los opcionales.

Los requerimientos quedan divididos en funcionales (la gran mayoría) y no funcionales (esbozados a nivel general). Los 
primeros están relacionados con: el sistema de clasificación; controles de acceso y seguridad; retención y disposición de registros; captura de documentos; búsqueda, recuperación y presentación de documentos; funciones administrativas y otras funcionalidades (por ejemplo: gestión de documentos no electrónicos, signaturas elec-

\begin{tabular}{|l|l|l|}
\hline Compañía & Tipo de organización & País \\
\hline Pfizer & Fabricante farmacéutico & UK \\
\hline Dera & Agencia de defensa & UK \\
\hline Hm Treasury & Gobierno central & UK \\
\hline Tower Technology & Proveedor de Erms & UK \\
\hline Technostock & Asesoría & España \\
\hline Ministry of Justice & Gobierno central & Italia \\
\hline
\end{tabular}
trónicas, etc.). En cuanto a los segundos, Tabla 1 están referidos a la siguientes cuestiones: facilidad de uso; funcionalidad, escalabilidad y disponibilidad del sistema; normas técnicas; requerimientos legales y normativos; sub-contratas y gestión de información a terceros y conservación a largo plazo y obsolescencia tecnológica.

\section{Functional requirements for electronic records management systems. Public Record Office (Reino Unido)}

Éste es un proyecto dirigido por el Public Record Office del Reino Unido en conjunción con la Central Computing and Telecommunications Agency (Ccta). En él han participado los departamentos y oficinas incluidos en la tabla 2. Actualmente, esta lista de requerimientos ha sido actualizada, y modificada de manera considerable, teniendo en cuenta (17):

-El e-Government interoperability framework (e$G I F)$ publicado por el UK Office of the e-Envoy.

- La especificación MoReq.

- La Data Protection Act 1998 y la Freedom of Information Act 2000.

- ISO 15489, nueva norma internacional de gestión de registros y su informe técnico.

Este documento está compuesto de 4 partes: el "enunciado de requerimientos funcionales" (functional requirements), una "norma de metadatos" sobre la base de los requerimientos establecidos (metadata standard), un "documento de referencia" (reference document) y, por último, una guía de implementación (implementation guide).

El listado de requerimientos queda estructurado en dos grandes secciones. Por un lado la sección A compuesta por los principales, y, por otro, la sección B que recoge los módulos de los opcionales. Ahora bien, en cada una de ellas se hace una distinción entre tres ti-

\begin{tabular}{|l|l|}
\hline Ministry of Defense & Court Service \\
\hline Hm Treasury & Crown Prosecution Service \\
\hline Inland Revenue & Treasury Solicitors Department \\
\hline Department of Health & Department of Social Security \\
\hline Medical Research Council & $\begin{array}{l}\text { Department of the Environment, Transport } \\
\text { and Regions }\end{array}$ \\
\cline { 1 - 1 } $\begin{array}{l}\text { Department for Education and } \\
\text { Employment }\end{array}$ & \\
\hline
\end{tabular}

pos: los obligatorios, los muy convenientes y los convenientes de cumplir.

La sección A queda subdividida a su vez en diez subcategorías que constituyen el grueso fundamental de la especificación en cuanto a los requerimientos relacionados con los siguientes puntos:

-A.1. Organización de los documentos, incluyendo la creación y mantenimiento del cuadro de clasificación.

-A.2. Captura, declaración y gestión de documentos.

-A.3. Búsqueda y presentación de documentos.

-A.4. Tareas de retención y disposición de documentos.

-A.5. Controles de acceso a los documentos y al sistema, incluyendo los marcados de control, los perfiles o roles de usuarios tanto individuales como de grupo, etc.

-A.6. Capacidad del sistema para llevar a cabo un registro de utilización ${ }^{5}$ de todas las acciones que se realicen sobre los documentos.

-A.7. Facultad que debe tener el sistema de redactar informes de distintos tipos sobre las actividades $\mathrm{y}$ estado en el que se encuentra.

-A.8. Facilidad de uso del sistema por parte de los usuarios que lo utilicen.

-A.9. Diseño y funcionamientos del sistema, teniéndose en cuenta aspectos tales como los interfaces, la escalabilidad, el almacenamiento, etc.

-A.10. Cumplimiento por parte del sistema de otras normas establecidas.

Por su parte, la sección B recoge tres módulos diferentes en un principio (los que listamos a continuación), ya que se tiene previsto ampliarla con otros tres nuevos (gestión de contenido, gestión de imágenes y workflow):

-B.1. Autentificación y codificación de documentos, incluyendo cuestiones acerca de los documentos que incluyen signaturas electrónicas o marcas de agua electrónicas.

-B.2. Gestión de documentos. 


\title{
Complete su colección de IWE/EPI
}

Deseo recibir las siguientes publicaciones: ejemplares de la Bibliografía IWE/EPI I992-200 I por correo postal impresos en papel (gratis).

la Bibliografía IWE/EPI 1992-200I por correo electrónico en formato PDF (gratis).

$\square$ álbumes de cd-rom con el texto íntegro de los primeros 100 números de la revista a 30 euros cada disco (uno es gratis para los suscriptores).

$\square$ ejemplares de los siguientes números atrasados de la revista (por favor, envíenme presupuesto).

Nombre:

Institución:

Dpto.: NIF institucional:

Dirección:

Código postal: Ciudad:

País:

Teléfono: Fax:

Correo-e:

Envíe este boletín por correo postal, fax o correo electrónico, a esta dirección:

\author{
Swets Blackwell \\ Caspe, 46 \\ 08010 Barcelona \\ España \\ Tel.: +34-932 70। 144; fax: 93270 I I45 \\ mnzang@es.swetsblackwell.com
}

-B.3. Gestión, en todo sus sentidos, de carpetas híbridas y físicas.

\section{System requirements for archiving electronics records. Public Record Office Victoria (Australia)}

Hablar del establecimiento de requerimientos en el Estado de Victoria, Australia, significa hablar de la primera especificación del Standard for the management of electronic records (Public Record Office Standard, Pros 99/007, versión 1.0, abril 2000)6.

A su vez, esta norma tendríamos que encuadrarla dentro de la Victorian Electronic Records Strategy $(\text { Vers })^{7}$, consistente en un marco de trabajo para el desarrollo de normas, guías y proyectos de implementación con el objetivo de conservar de forma segura y auténtica los documentos electrónicos creados y mantenidos por el Gobierno de Victoria (18).

En definitiva, se trata de la solución propuesta por este estado, auspiciada por el Public Record Office
Victoria (Prov), para la gestión de documentos electrónicos.

A grandes rasgos, la Vers pretende:

-Especificar un marco de trabajo mínimo y simple para la gestión de documentos electrónicos.

- Definir un formato a largo plazo para la captura de los documentos que necesitan ser guardados por un largo período de tiempo.

-Asegurar que todos los documentos sean almacenados en un formato a largo plazo, para hacer posible su visualización en un futuro independientemente del sistema que los creó.

-Especificar métodos para automatizar la captura de documentos desde los sistemas de las oficinas.

- Detallar el modo y las formas de capturar información sobre los documentos y encapsularla con éstos para asegurar que en el futuro sean entendidos en su contexto. 
-Especificar un método para la seguridad de los documentos de tal manera que se detecten los cambios no autorizados.

La Pros 99/007 detalla los principales requerimientos para la gestión y conservación de documentos electrónicos en el sector público de Victoria. Incluye tres especificaciones:

a. Pros 99/007. Specification 1. System requirements for archiving electronic records: lista los requerimientos que deben cumplir los sgdes, recogidos en tres apartados:

- Captura.

-Gestión; incluye cuestiones tales como control de acceso, retención y disposición, transferencias, etc.

- Recuperación, es decir, relacionados con el interfaz de usuario, la búsqueda, la presentación de los resultados, etc.

b. Pros 99/007. Specification 2. Vers metadata scheme: recoge los metadatos necesarios para una adecuada gestión y conservación de los documentos electrónicos.

c. Pros 99/007. Specification 3. Vers standard electronic record format: proporciona detalles técnicos sobre la conservación de los documentos electrónicos a largo plazo mediante la utilización del Vers long term format, también conocido como Vers encapsulated object (VEO). También recoge cuestiones relacionadas con el uso de signaturas digitales.

\section{Design criteria standard for electronic records management. Software applications. Department of Defense (EUA)}

Se trata de una norma desarrollada en el marco de la Directiva 5015.2 Department of Defense Records Management Program ${ }^{8}$ de 6 de marzo de 2000, que proporciona una guía de implementación y procedimiento de gestión de documentos en el Departamento de Defensa de los EUA. Es de uso obligatorio para llevar a cabo la implementación de programas de gestión de documentos en todos los componentes ${ }^{9}$ del $D o D$ (19). El texto, en formato pdf, que se nos ofrece en la web $^{10}$ es una versión revisada de la $D o D$ 5015.2-STD de junio de 2002, que viene a cancelar la $D o D$ 5015.2STD Design criteria standard for electronic records management. Software applications de 24 de noviembre de 1997.

El grueso principal de la norma se divide en 4 capítulos:
- Información general. Aquí quedan recogidos de forma muy escueta los objetivos y limitaciones de la norma.

-Requerimientos obligatorios. Se incluyen, divididos en generales y detallados, aquellos que deben cumplir obligatoriamente los sgdes.

-Requerimientos no-obligatorios (opcionales). Se dividen en los definidos por las actividades de adquisición y uso, y otros útiles para la aplicación de gestión de documentos.

- Gestión de documentos clasificados. Requerimientos necesarios para llevar a cabo la gestión de documentos clasificados y otros de seguridad opcionales.

\section{Conclusiones}

El desarrollo de un listado de requerimientos funcionales como paso previo al diseño e implementación de un sgde resulta de vital importancia, pues constituye la base o espina dorsal sobre la cual va a ser montado. Las grandes áreas en esta materia son:

-Configuración y estructura del cuadro de clasificación y su mantenimiento.

-Controles y gestión de acceso por medio del establecimiento de categorías de seguridad y realización de copias de seguridad.

-Retención y disposición de documentos, teniéndose en cuenta la transferencia, exportación y destrucción de registros.

- Captura de los distintos tipos de documentos, independientemente de que ésta se haga de forma individual o en masa.

- Técnicas de búsqueda y recuperación de documentos y las distintas formas de presentación de los resultados.

Aunque éstas son las áreas de requerimientos principales establecidas en todas las listas, debemos anotar también que algunas de ellas coinciden en recoger otros igualmente importantes a nuestro parecer. Nos referimos a los relacionados con:

-Funciones administrativas generales del sistema como restablecimientos o recuperación desde copias de seguridad, administración y gestión de usuarios, control del espacio de almacenamiento, redacción de informes acerca de distintos aspectos tales como el número de ficheros, actividades por usuario, etc.

- Gestión de documentos no electrónicos o físicos permitiéndose retener y disponer estos ficheros o carpetas híbridas.

- Gestión del correo electrónico, tanto de la documentación que sale como de la que entra. 


\begin{tabular}{|c|c|}
\hline $\begin{array}{c}\text { MoReq } \\
\text { Requerimientos funcionales } \\
\text { Nombre del requerimiento }\end{array}$ & Resultados \\
\hline 3. Sistema de clasificación. & \\
\hline $\begin{array}{l}\text { 3.1. Configuración del sistema de } \\
\text { clasificación. } \\
\text { 3.2. Clases y ficheros. } \\
\text { 3.3. Volúmenes. } \\
\text { 3.4. Mantenimiento del sistema de } \\
\text { clasificación. }\end{array}$ & $\begin{array}{l}\text { Todas las listas de requerimientos estudiadas } \\
\text { contienen requerimientos relacionados con el } \\
\text { sistema de clasificación y s u mantenimiento } \\
\text { excepto la lista del Prov. } \\
\text { Este hecho resulta curioso pues este tipo de } \\
\text { requerimientos es considerado principal u } \\
\text { obligatorio tanto para MoReq como para la lista } \\
\text { del } P R O(\mathrm{UK}) \text { y del } D o D \text {. }\end{array}$ \\
\hline \multicolumn{2}{|l|}{ 4. Controles y seguridad. } \\
\hline 4.1. Acceso. & $\begin{array}{l}\text { Todas las listas incluyen requerimientos } \\
\text { relacionados con el control del acceso. }\end{array}$ \\
\hline 4.2. Registros de utilización. & $\begin{array}{l}\text { Aunque todas las listas incluyen requerimientos } \\
\text { relacionados con los registros de utilización, la } \\
\text { forma de referirse a él varía de una a otra. }\end{array}$ \\
\hline $\begin{array}{l}\text { 4.3. Copias de seguridad y } \\
\text { recuperación. }\end{array}$ & $\begin{array}{l}\text { La lista del } P R O \text { (UK) abarca este tipo de } \\
\text { requerimientos de forma muy sucinta. } \\
\text { Por el contrario, la lista del } D O D \text { lo abarca de } \\
\text { forma un poco más amplia y los incluye dentro de } \\
\text { los requerimientos obligatorios. }\end{array}$ \\
\hline $\begin{array}{l}\text { 4.4. Historial de los movimientos del } \\
\text { registro. }\end{array}$ & $\begin{array}{l}\text { No son incluidos en ninguna de las listas. } \\
\text { Consideramos este asunto de gran utilidad como } \\
\text { mecanismo de control tanto de la localización } \\
\text { actual de los documentos como los movimientos o } \\
\text { cambios de localización de los mismos. }\end{array}$ \\
\hline 4.5. Autenticidad. & $\begin{array}{l}\text { Sólo la lista Provr ecoge requerimientos } \\
\text { relacionados con la autenticidad de los } \\
\text { documentos una vez que éstos son capturados; y } \\
\text { lo hace en términos de mantener la integridad de } \\
\text { la evidencia. }\end{array}$ \\
\hline 4.6. Categorías de seguridad. & $\begin{array}{l}\text { Los requerimientos relacionados con las } \\
\text { categorías de seguridad como mecanismo para } \\
\text { limitar aún más el acceso a I os registros son } \\
\text { recogidos solamente en la lista del } P R O(\mathrm{UK}) \text {. } \\
\text { Exactamente hace referencia a los marcados de } \\
\text { control. }\end{array}$ \\
\hline \multicolumn{2}{|l|}{ 5. Retención y disposición. } \\
\hline 5.1. Calendario de retención. & $\begin{array}{l}\text { Todas las listas recogen requerimientos } \\
\text { relacionados con los calendarios o tablas de } \\
\text { retención/disposición. }\end{array}$ \\
\hline 5.2. Revisión. & $\begin{array}{l}\text { Sólo la lista del } P R O \text { (UK) recoge requerimientos } \\
\text { relacionados con los procesos de revisión o } \\
\text { chequeo de los documentos una vez que han } \\
\text { alcanzado la fecha de retención. }\end{array}$ \\
\hline $\begin{array}{l}\text { 5.3. Transferencia, exportación y } \\
\text { destrucción. }\end{array}$ & Quedan recogidos en todas las listas. \\
\hline \multicolumn{2}{|l|}{ 6. Captura de registros. } \\
\hline 6.1. Captura. & $\begin{array}{l}\text { Al igual que en el caso anterior, están incluidos en } \\
\text { todas las listas. }\end{array}$ \\
\hline
\end{tabular}




\begin{tabular}{|c|c|}
\hline 6.2. Importación en masa. & $\begin{array}{l}\text { Los requerimientos relacionados con la capacidad } \\
\text { de aceptar la I legada de documentos en masa } \\
\text { sólo quedan recogidos en la lista del } P R O \text { (UK). } \\
\text { Resulta importante incluir prestaciones para } \\
\text { gestionar un proceso de captura de estas } \\
\text { características. }\end{array}$ \\
\hline 6.3. Tipos de documentos. & $\begin{array}{l}\text { Los requerimientos relacionados con la captura de } \\
\text { un rango de diversos tipos de documentos } \\
\text { quedan recogidos sólo en la lista del } P R O \text { (UK), } \\
\text { aunque lo hacen de forma muy sucinta. }\end{array}$ \\
\hline 6.4. Gestión del correo electrónico. & $\begin{array}{l}\text { Los requerimientos relacionados con la capacidad } \\
\text { de capturar los mensajes de correo electrónico } \\
\text { tanto enviados como recibidos y s us ficheros } \\
\text { adjuntos quedan recogidos en las listas del } D o D \text { y } \\
\text { del } P R O(\mathrm{UK}) \text {. }\end{array}$ \\
\hline 7. Referenciar/hacer referencia a. & $\begin{array}{l}\text { Los requerimientos relacionados con la asignación } \\
\text { de identificadores únicos a las distintas entidades } \\
\text { del sistema quedan recogidos en todas las listas } \\
\text { excepto la del } P R O \text { (UK). }\end{array}$ \\
\hline $\begin{array}{l}\text { 8. Búsqueda, recuperación y } \\
\text { presentación. }\end{array}$ & \\
\hline 8.1. Búsqueda y recuperación. & $\begin{array}{l}\text { Los requerimientos relacionados con la búsqueda } \\
\text { y recuperación de documentos quedan recogidos } \\
\text { en todas las listas. }\end{array}$ \\
\hline $\begin{array}{l}\text { 8.2. Presentación: presentación de } \\
\text { registros en pantalla. }\end{array}$ & \multirow{3}{*}{$\begin{array}{l}\text { Ahora bien, el tema de los distintos tipos de } \\
\text { presentación de los resultados de búsqueda sólo } \\
\text { queda recogido, de forma muy escueta, en la lista } \\
\text { del } P R O \text { (UK). }\end{array}$} \\
\hline 8.3. Presentación: impresión. & \\
\hline 8.4. Presentación: otros. & \\
\hline \multicolumn{2}{|l|}{ 9. Funciones administrativas. } \\
\hline 9.1. Administración general. & $\begin{array}{l}\text { Sólo la I ista del Prov no recoge requerimientos } \\
\text { relacionados con la administración general del } \\
\text { sistema (restablecimiento de copias de seguridad, } \\
\text { administración de usuarios, capacidad de realizar } \\
\text { cambios globales, etc.). }\end{array}$ \\
\hline 9.2. Presentación de informes. & $\begin{array}{l}\text { Las listas del } D o D \text { y del } P R O(\mathrm{UK}) \text { recogen } \\
\text { requerimientos relacionados con la capacidad de } \\
\text { redactar informes. }\end{array}$ \\
\hline $\begin{array}{l}\text { 9.3. Cambio, eliminación y redacción } \\
\text { de documentos. }\end{array}$ & $\begin{array}{l}\text { Sólo la lista del Prov recoge requerimientos } \\
\text { relacionados con la eliminación de do cumentos. } \\
\text { Sin embargo no contempla nada a cerca de la } \\
\text { realización de cambios y redacción de extractos } \\
\text { de documentos. }\end{array}$ \\
\hline \multicolumn{2}{|l|}{ 10. Otra funcionalidad. } \\
\hline $\begin{array}{l}\text { 10.1. Gestión de documentos no } \\
\text { electrónicos. }\end{array}$ & $\begin{array}{l}\text { Los requerimientos para la gestión de documentos } \\
\text { físicos quedan recogidos en todas las listas } \\
\text { excepto la del Prov. }\end{array}$ \\
\hline $\begin{array}{l}\text { 10.2. Retención y disposición del } \\
\text { fichero híbrido. }\end{array}$ & $\begin{array}{l}\text { Por otro lado, los requerimientos relacionados con } \\
\text { la retención y disposición de los ficheros híbridos } \\
\text { (registros físicos y electrónicos) sólo quedan } \\
\text { recogidos en la lista del } P R O(\mathrm{UK}) \text {. }\end{array}$ \\
\hline
\end{tabular}


Tabla 3 (continuación)

\begin{tabular}{|c|c|}
\hline & \multirow[b]{2}{*}{$\begin{array}{l}\text { La lista del } D O D \text { hace referencia a los } \\
\text { requerimientos de gestión de documentos y } \\
\text { prestaciones de workflow de manera conjunta y } \\
\text { de forma muy reducida. } \\
\text { Por su parte, la lista del } P R O \text { (UK) también recoge } \\
\text { requerimientos relacionados con la gestión de } \\
\text { documentos pero de forma poco desarrollada. } \\
\text { Además, tiene planificado introducir } \\
\text { requerimientos sobre workflow en un módulo } \\
\text { opcional. } \\
\text { Por tanto los requerimientos relacionados con el } \\
\text { workflow podemos decir que prácticamente no } \\
\text { son tratados en ninguna de las especificaciones. } \\
\text { No obstante, sería de gran importancia que se } \\
\text { tuvieran en cuenta en los sistemas de grandes } \\
\text { organizaciones que lo utilicen. }\end{array}$} \\
\hline $\begin{array}{l}\text { 10.3. Gestion de documentos. } \\
\text { 10.4. Organización del trabajo } \\
\text { (workflow). }\end{array}$ & \\
\hline 10.5. Signaturas electrónicas. & $\begin{array}{l}\text { Los requerimientos relacionados con el uso de } \\
\text { signaturas electrónicas como mecanismos de } \\
\text { autentificación de documentos quedan recogidos } \\
\text { en todas las listas excepto en la del } D o D \text {. } \\
\text { Resulta curioso como en la lista del Provse hace } \\
\text { especial hincapié, en sus tres bloques de } \\
\text { requerimientos, en el tema de las signaturas } \\
\text { electrónicas. }\end{array}$ \\
\hline 10.6. Codificación. & $\begin{array}{l}\text { Los requerimientos relacionados con la } \\
\text { codificación sólo son recogidos en la lista del } P R O \\
\text { (UK). }\end{array}$ \\
\hline $\begin{array}{l}\text { 10.7. Marcas de agua, filigranas } \\
\text { electrónicas, etc. }\end{array}$ & $\begin{array}{l}\text { Ninguna lista recoge requerimientos relacionados } \\
\text { con el establecimiento de marcas de agua, } \\
\text { filigranas electrónicas, etc. }\end{array}$ \\
\hline $\begin{array}{l}\text { 10.8. Interoperatividad y apertura } \\
\text { (openness). }\end{array}$ & $\begin{array}{l}\text { Sólo la lista del DoD recoge requerimientos } \\
\text { relacionados con la interoperatividad entre } \\
\text { sistemas o aplicaciones. }\end{array}$ \\
\hline \multicolumn{2}{|l|}{ Requerimientos no funcionales } \\
\hline \multicolumn{2}{|l|}{ Nombre del requerimiento } \\
\hline 11.1. Facilidad de uso. & $\begin{array}{l}\text { Todas las listas de requerimientos recogen de } \\
\text { forma más o menos detallada requerimientos } \\
\text { relacionados facilitar el uso del sistema a los } \\
\text { usuarios. }\end{array}$ \\
\hline 11.2. Funcionamiento y escalabilidad. & \multirow{2}{*}{$\begin{array}{l}\text { Tanto la lista del } P R O(\mathrm{UK}) \text { como la del } D o D \\
\text { recogen requerimientos relacionados con el } \\
\text { funcionamiento del sistema, aunque sólo esta } \\
\text { última recoge requerimientos relacionados con la } \\
\text { disponibilidad del mismo. }\end{array}$} \\
\hline 11.3. Disponibilidad del sistema. & \\
\hline 11.4. Normas técnicas. & \multirow{2}{*}{$\begin{array}{l}\text { Como en el caso anterior, tanto la lista del } P R O \\
\text { (UK) como la del } D O D \text { recogen requerimientos } \\
\text { relacionados con las normas técnicas y } \\
\text { requerimientos legales y n ormativos a los que } \\
\text { debe atenerse el sistema. }\end{array}$} \\
\hline $\begin{array}{l}\text { 11.5. Requerimientos legales y } \\
\text { normativos. }\end{array}$ & \\
\hline $\begin{array}{l}\text { 11.6. Sub contratas y gestión de } \\
\text { información a terceros. }\end{array}$ & $\begin{array}{l}\text { Ninguna de las listas recoge requerimientos } \\
\text { relacionados con el outsourcing. }\end{array}$ \\
\hline $\begin{array}{l}\text { 11.7. Conservación a largo plazo y } \\
\text { obsolescencia tecnológica. }\end{array}$ & $\begin{array}{l}\text { Los requerimientos relacionados con la } \\
\text { conservación no son recogidos de forma expresa } \\
\text { en ninguna de las especificaciones. } \\
\text { Ahora bien en la lista del Prov y en la del } D o D \text {, se } \\
\text { recogen requerimientos que podríamos asemejar } \\
\text { a éstos. }\end{array}$ \\
\hline
\end{tabular}


-Aspectos no funcionales como: facilidad de uso del sistema (interfaz de usuario), funcionamiento, escalabilidad y disponibilidad del mismo y normas técnicas y requerimientos legales y normativos a los que debe atenerse.

Ahora bien, si realizamos una comparación de la similitud de cada una de las listas estudiadas con respecto a MoReq (especificación de ámbito europeo) sacamos los resultados presentados en la tabla 3 .

\section{Notas}

1. El conjunto de normas australianas sobre gestión de documentos es: $A S$ 4390.1-1996, AS 4390.2-1996, AS 4390.3-1996, AS 4390.4-1996, AS 4390.5-1996 y AS 4390.6-1996.

2. Marc Fresko y Martin Waldron. El director del proyecto fue Keith Cornwell (managing director de Cornwell Affiliates plc.), y el encargado del proyecto en la Comisión Europea fue Paul E. Murphy.

3. Cornwell Affiliates plc. es la empresa seleccionada por la Comisión Europea para desarrollar la especificación MoReq. Esta empresa británica presta servicios de asesoría en materia de gestión y tecnologías de la información y, entre ellos se encuentra el de asesoría en gestión de registros electrónicos.

http://www.cornwell.co.uk/index.html

4. Francisco Barbedo (Arquivo Distrital do Porto, Portugal), Keith Batchelor (consultor independiente, Reino Unido), Nils Brübach (Archives School, Marburg, Alemania), Miguel Camacho (Sadiel S. A., España), Luciana Duranti (School of Library and Information Studies, University of British Columbia, Canadá), Mariella Guercio (Università di Urbino, Istituto di Studi Beni Archivistici e Librari, Italia), Peter Horsman (Netherlands Institute for Archival Education and Research, Holanda) y JeanPierre Teil (Archives Nationales, Francia).

5. Se trata de información sobre todas las transacciones u otras actividades que han afectado o cambiado entidades (elementos metadatos, registros, ficheros) que contiene suficiente detalle como para permitir reconstruir una actividad previa.

6. Esta norma puede ser consultada electrónicamente en dos formatos. Consultado en: 08-07-02.

http://www.prov.vic.gov.au/vers/standards/pros9907/99-7toc.htm

http://www.prov.vic.gov.au/vers/standards/pros9907/99-7.pdf

7. Vers. Consultado en: 08-07-02.

http://vers.imagineering.net.au/site-ver2/

8. Más información acerca de esta directiva. Consultado en: 18-07-02. http://www.dtic.mil/whs/directives/corres/pdf/d50152_030600/d50152p.pd $f$

9. Los componentes del $D o D$ son los siguientes: la Office of the Secretary of Defense, los Military Departments, el Chairman of the Joint Chiefs of Staff, los Combatant Commands, el Inspector General of the Department of Defense, las Defense Agencies, las DoD Field Activities y otras entidades dentro del $D o D$.

10. Joint interoperability test command records management application (RMA): DoD 5015.2-STD.

http://jitc.fhu.disa.mil/recmgt/standards.htm

\section{Referencias bibliográficas}

1) Peis Redondo, Eduardo. "Archivando registros electrónicos". En: $V$ Jornadas de archivos electrónicos, 2002, pp. 21-31.

2) Pittsburgh Electronic Records Project. Functional requirement for evidence in recordkeeping. Consultado en: 22-01-02.

http://www.lis.pitt.edu/ nhprc/progl.html

3) Department of Defense of the United States. Design criteria standard for electronic records management software applications. Consultado en: 18-07-02.

http://jitc.fhu.disa.mil/recmgt/p50152s2.pdf
4) The National Archives of Australia. Designing and implementing recordkeeping systems. Consultado en: 22-01-02.

http://www.naa.gov.au/recordkeeping/dirks/summary.html

5) National Archives of Norway. Noark-4: Norwegian recordkeeping system. Version 4. Consultado en: 22-01-02.

http://www.riksarkivet.no/noark-4/noark-eng.pdf

6) Canadian State Archives. Recordkeeping in the electronic work environment. Consultado en: 22-01-02.

http://www.archives.ca/06/0603_e.html

7) State Archives of Victoria (Australia). System requirements for archiving electronic records. Consultado en: 22-01-02.

http://www.prov.vic.gov.au/vers/standard/99-7-1toc.htm

8) Delaware State Archives. Model guidelines for electronic records. Consultado en: 22-01-02.

http://www.archives.lib.de.us/recman/g-lines.htm

9) New York State Archives. Functional requirements to ensure the creation, maintenance, and preservation of electronic records. Consultado en: 22-01-02.

http://www.ctg.albany.edu/resources/abstract/mfa-4.html

10) Kansas State Historical Society. Kansas electronic records management guidelines. Consultado en: 22-01-02.

http://www.kshs.org/archives/recmgt.htm

11) University of British Columbia. The preservation of the integrity of electronic records. Consultado en: 22-01-02.

http://www.slais.ubc.ca/users/duranti/

12) Indiana University. Indiana University electronic records project. Functional requirements for recordkeeping systems. Consultado en: 2201-02.

http://www.indiana.edu/ libarch/funcreqs.html

13) European Commission. Model requirements for the management of electronic records. Luxembourg: Office for Official Publications of the European Communities, 2002. Isbn 92-894-1290-9.

14) ISO. International Standard ISO 15489: information and documentation-Records management. Switzerland: ISO, 2001.

15) Bustelo Ruesta, Carlota. "Los sistemas de gestión electrónica de la documentación y la teoría del ciclo vital de los documentos en las organizaciones". En: Scire, 1997, v. 3, n. 2, pp. 45-53.

16) Kansas electronic records management guidelines. Consultado en: 28-06-02.

http://www.kshs.org/archives/ermguide.htm

17) Requirements for electronic records management systems. 1 : functional requirements. Consultado en: 5-11-02.

http://www.pro.gov.uk/recordsmanagement/eros/invest/2002requirementsfi nal.pdf

18) Public Record Office Victoria. Victorian electronic records strategy. Consultado en: 08-07-02.

http://www.prov.vic.gov.au/vers/welcome.htm

19) Department of Defense of the United States. Design criteria standard for electronic records management software applications. Consultado en: 18-07-02.

http://jitc.fhu.disa.mil/recmgt/p50152s2.pdf

David Gómez Domínguez; Antonio A. Ruiz Rodríguez;

Eduardo Peis Redondo, Facultad de Biblioteconomía y Documentación, Universidad de Granada.

dgomez@fedro.ugr.es

aangel@ugr.es

epeis@ugr.es 